\title{
Perioperative Care of a Patient with Refractory Idiopathic Thrombocytopenic Purpura Undergoing Total Knee Arthroplasty
}

\author{
Rohit Singhal, MS (Ortho), MRCSEd ${ }^{1}$, Veera Gudimetla, FRCA ${ }^{2}$, Andrew Stewart, FRCP Edin, FRCPath ${ }^{3}$, \\ Karen L Luscombe, FRCS (Tr \& Orth) ${ }^{1}$ and Charalambos P Charalambous, MSc, MD, FRCS (Tr \& Orth) ${ }^{4}$ \\ ${ }^{1}$ Department of Trauma and Orthopaedics, Leighton Hospital, Crewe; ${ }^{2}$ Department of Anaesthesiology, Leighton Hospital, Crewe; ${ }^{3}$ Department of Haematology, \\ University Hospital of North Staffordshire, Stoke-on-Trent; ${ }^{4}$ Department of Trauma and Orthopaedics, Blackpool Victoria Hospital, Blackpool, United Kingdom
}

Idiopathic thrombocytopenic purpura (ITP) is an autoimmune disorder leading to low platelet count and an increased risk of bleeding. Major joint replacement surgery in a patient with ITP can be associated with severe postoperative bleeding. We present our experience of perioperative management in a patient with severe refractory chronic idiopathic thrombocytopenic purpura who successfully underwent a cemented total knee replacement.

Key words: Perioperative management, Immune thrombocytopenic purpura, Total knee replacement.

\section{Introduction}

Refractory chronic immune thrombocytopenic purpura (ITP) is an auto-immune disorder with persistently low platelet count which is unresponsive to any recognised modality of treatment without unacceptable toxicity ${ }^{1}$. Surgical procedures carried out in patients with ITP involve risk of severe intra operative and postoperative bleeding and infection. Appropriate preoperative preparation of patients with severe ITP undergoing major joint replacement surgery is essential to avoid such complications.

We present a case report of a 61-year-old male patient with severe chronic refractory ITP who underwent cemented total

Received August 20, 2012; Revised September 21, 2012;

Accepted October 24, 2012.

Correspondence to: Rohit Singhal, MS (Ortho), MRCSEd.

Department of Trauma and Orthopaedics, Leighton Hospital, 6,

Tennyson Drive, Ormskirk, United Kingdom.

Tel: +44-7838008900, Fax: +44-1250768999

Email: singhal.rohit75@gmail.com

This is an Open Access article distributed under the terms of the Creative Commons Attribution Non-Commercial License (http://creativecommons.org/licenses/by-nc/3.0/) which permits unrestricted non-commercial use, distribution, and reproduction in any medium, provided the original work is properly cited. knee replacement with careful and meticulous perioperative management.

\section{Case Report}

A 61-year-old man with a history of ITP presented with chronic severe right knee pain. He had been suffering from ITP for eleven years and despite corticosteroids, dapsone and azathioprine

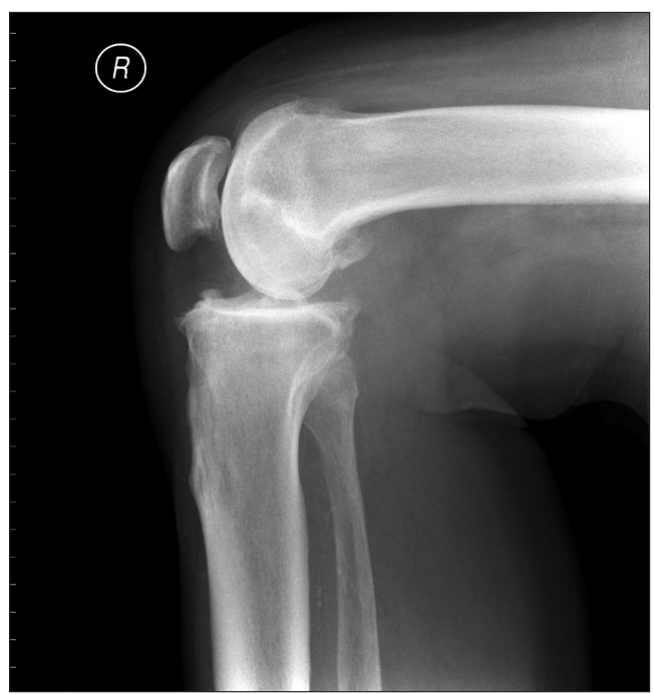

Fig. 1. Preoperative lateral radiograph of right knee. 


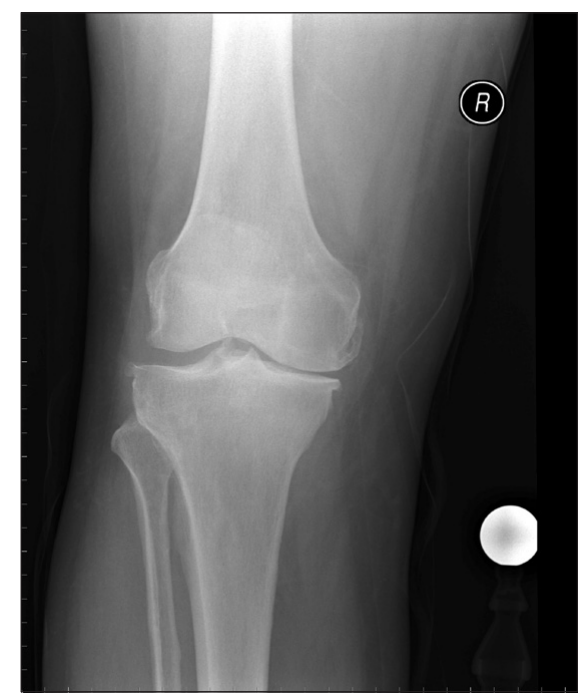

Fig. 2. Preoperative Antero-posterior (AP) radiograph of right knee.

treatment as well as previous splenectomy his platelet count remained in the range $20-30 \times 10^{9} / \mathrm{L}$ with occasional dips to $<10 \times 10^{9}$.

Clinical examination of the knee revealed a correctable varus deformity of five degrees with no fixed flexion deformity and knee flexion of ninety degrees. The skin quality around the knee was satisfactory with no distal neuro-vascular deficit. Radiographs of the knee (Figs. 1,2) confirmed severe osteoarthritis with absent joint space in the medial compartment of the right knee. Considering the severe changes on the X-rays and effect on his quality of life a decision to undertake a right sided cemented knee replacement was made. The possible risks involved were discussed at length with the patient by both the orthopaedic and haematology teams. After careful consideration he consented to proceed with the operation.

The patient received $28 \mathrm{~g}$ of intravenous immunoglobulin (IV Ig) per day (equivalent to $400 \mathrm{mg} / \mathrm{kg}$ for a $70 \mathrm{~kg}$ person) for five days prior to the knee replacement. A platelet count of $280 \times 10^{9} / \mathrm{L}$ was achieved and it was deemed safe to carry out the procedure. As the patient had been receiving prednisolone at a dose of 10 $\mathrm{mg}$ daily for some months prior to the operation, hydrocortisone $150 \mathrm{mg}$ was administered on the day of operation, according to the advice by the anaesthetic team. The operation was conducted under general anaesthesia with sciatic and femoral nerve blocks. Cemented total knee replacement was performed using midline skin incision with medial parapatellar approach. Cruciate retaining PFC sigma (DePuy Orthopaedics Inc, Warsaw, IN, USA) implant was used with Palacos cement (Heraeus, Wehrheim, Germany). Meticulous tissue handling was observed

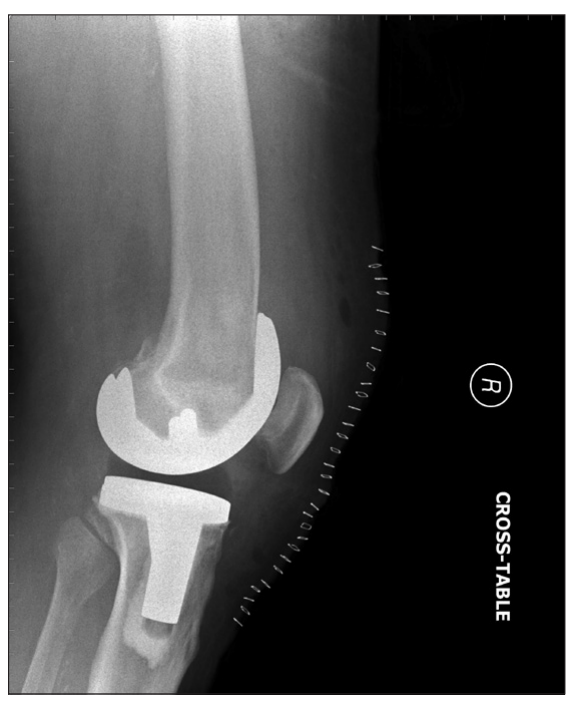

Fig. 3. Postoperative lateral radiograph of the right knee.

throughout the whole procedure. No alterations were made to the routinely used surgical technique and no drains were used at the end of the procedure. No additional blood conservation measures such as haemovac or cell saver were used. No particular difficulties were faced during the operation. A total tourniquet time of 75 minutes was noted.

Postoperatively, the patient was particularly observed for excessive bleeding from the operative wound and his platelet count was monitored daily. Low molecular weight heparin which is routinely recommended for prevention of venous thromboemobolism in patients undergoing major joint replacement surgery, was not prescribed due to high likelihood of postoperative bleeding in this case. Also, non steroidal anti-inflammatory drugs which are known to cause platelet dysfunction were avoided. Instead, mechanical thromboprophylaxis was used with anti-embolism stocking sand intermittent calf compression pumps.

On postoperative day one platelet count was $140 \times 10^{9}$, on the second day platelet count dropped down to $137 \times 10^{9}$ and on the third postoperative day it was $73 \times 10^{9}$. In view of this, 28 $\mathrm{g}(400 \mathrm{mg} / \mathrm{kg})$ of IV Ig per day was commenced on the third postoperative day for another three days after discussion with the haematology team. At this stage, low molecular weight heparin was commenced to counter the hypercoagulability state known to be caused by intravenous immunoglobulins ${ }^{2}$. His platelet count dropped down to $37 \times 10^{9}$ on postoperative day four. On day seven the platelet count improved to $190 \times 10^{9}$.

Routine postoperative ambulation was carried out. The patient was mobilised with the aid of crutches initially. He was 


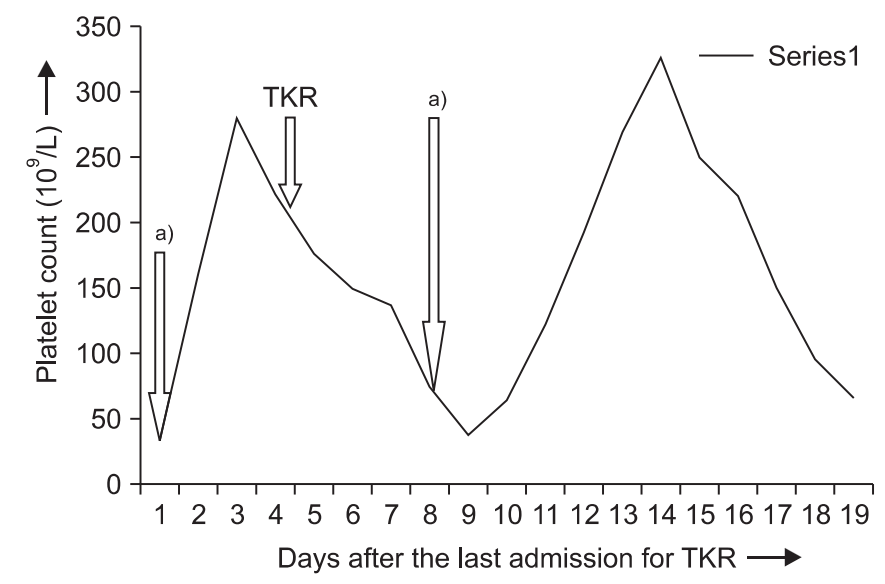

Fig. 4. Trend in the Platelet count after the admission for knee arthroplasty procedure and major intervening therapeutic events. TKR, total

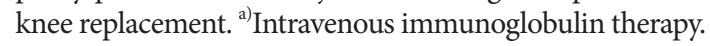

discharged from the hospital on the eighth postoperative day after achieving independent walking with stair climbing and knee flexion of 80 degrees.

The patient was reviewed in the haematology clinic three days following discharge from the hospital. The platelet count measured then was $327 \times 10^{9}$. His platelet count trend is shown in Fig. 3. He was reviewed in the orthopaedic clinic after one week, three week and six weeks. The postoperative radiographs of his knee were satisfactory (Figs. 4, 5). No complications were observed. At three months follow-up $130^{\circ}$ of knee flexion was achieved. His postoperative Oxford Knee score was calculated to be 40 . The patient was able to carry out all the activities of daily living including driving and he was very satisfied with the outcome.

\section{Discussion}

This report describes a case of severe refractory chronic ITP undergoing cemented total knee replacement surgery for severe osteoarthritis of the knee. Successful result was achieved with constant supervision, careful monitoring and multi-disciplinary team approach.

ITP is an immune disorder characterised by auto antibodies formation against platelets leading to accelerated platelet destruction and retarded platelet production ${ }^{1)}$. The prevalence of ITP in adults in the UK is approximately 5.8 to 6.6 per 100,000. As compared to ITP in children, ITP in adults often runs a chronic course and in about $10 \%$ of adult patients are refractory to treatment $t^{3)}$. The risk of bleeding requiring treatment increase with increasing severity of thrombocytopenia and is highest in patients with platelet count of $<20 \times 10^{9} / \mathrm{L}^{4}$. Treatment is seldom

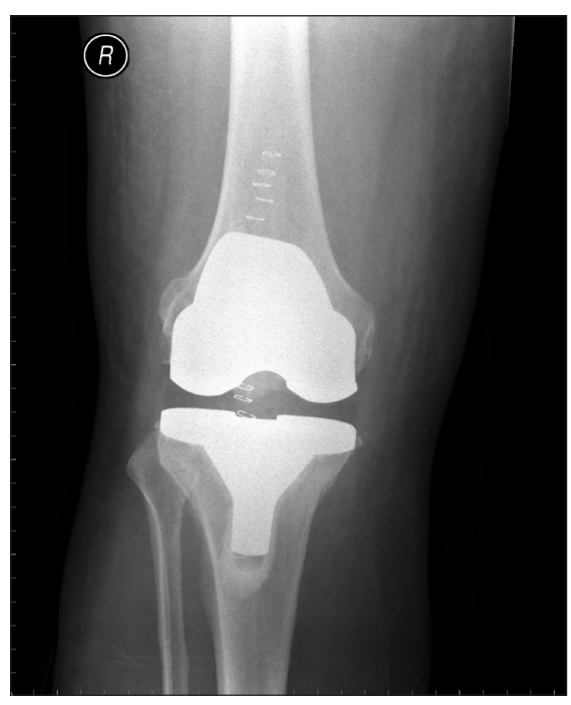

Fig. 5. Postoperative antero-posterior radiograph of right knee.

required with the platelet count of more than $30 \times 10^{9} / \mathrm{L}$ unless the patient is undergoing a surgical procedure. Platelet count of more than $80 \times 10^{9} / \mathrm{L}$ is the recommended level for undertaking major surgery ${ }^{5}$. Treatment options are categorized into first line and second line therapy. First line includes oral steroid therapy and pooled human immunoglobulins. Second line therapy includes splenectomy, intravenous anti-D, androgen therapy (Danazol) and immunosuppressive drugs such as vinca alkaloids, azathioprine, cyclosporine A and rituximab. High dose intravenous immunoglobulins along with coticosteroids have been shown to stimulate early response which might be particularly useful in surgical patients $s^{5)}$.

Many cases with ITP undergoing cardio vascular surgery ${ }^{6}$ and cancer surgery ${ }^{7)}$ have been reported but limited case reports of such patients undergoing major orthopaedic operations have been described. Kim et al. ${ }^{8)}$ described a series of five patients undergoing total hip replacement for osteonecrosis of femoral head related to underlying ITP. Most of the patients described in this article were less than 60 year of age at the time of surgery.

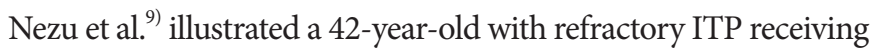
hip replacement under the cover of vinca alkaloids and colchicine as the platelet count could not be elevated by the first line and second line therapy. However, to our knowledge, no case of total knee replacement in patient with ITP has been described in the literature and there are no specific guidelines issued regarding the perioperative management of such patients.

Patients older than 60 years and those with previous history of bleeding are at higher risk of complications ${ }^{4}$. Post operative intra-articular warrants special consideration in cases of knee replacement as compared to hip replacement because of the risk 
of arthrofibrosis due to bleeding leading to knee stiffness. Bone cuts made during knee replacement surgery results in large raw surfaces of bone with high potential of bleeding. In the present case, cement was used to cover the bleeding bony surfaces as cemented knee replacement has been shown to result in lower perioperative blood loss as compared to uncemented knee replacement ${ }^{10)}$.

The clinical needs and perioperative management of patients undergoing an emergency operation are different from that of those undergoing elective surgery such as a joint replacement procedure, which can be deferred for indefinite period as the procedure is not life saving. Surgeons might postpone knee or hip replacement, which is mainly undertaken to improve the quality of life, if the expected intra or post operative complications are deemed to be life threatening. With specific guidelines, scrupulous preparation, close communication and constant monitoring these procedures can be conducted with a successful outcome.

ITP is a complex disorder. Close monitoring is paramount to prevent severe complications. Medical therapy and appropriate surgical technique can minimise complications in patients with severe refractory ITP undergoing total knee replacement.

\section{References}

1. Chouhan JD, Herrington JD. Treatment options for chronic refractory idiopathic thrombocytopenic purpura in adults: focus on romiplostim and eltrombopag. Pharmacotherapy. 2010;30:666-83.

2. Iroh Tam PY, Richardson M, Grewal S. Fatal case of bilateral internal jugular vein thrombosis following IVIg infusion in an adolescent girl treated for ITP. Am J Hematol. 2008;83:323-5.
3. George JN. Management of patients with refractory immune thrombocytopenic purpura. J Thromb Haemost. 2006;4:1664-72.

4. Portielje JE, Westendorp RG, Kluin-Nelemans HC, Brand A. Morbidity and mortality in adults with idiopathic thrombocytopenic purpura. Blood. 2001;97:2549-54.

5. British Committee for Standards in Haematology General Haematology Task Force. Guidelines for the investigation and management of idiopathic thrombocytopenic purpura in adults, children and in pregnancy. Br J Haematol. 2003;120:574-96.

6. Rossi M, Lewis M, Hutchinson N. Coronary artery bypass grafting in idiopathic thrombocytopenia: use of thromboelastometry without platelet transfusion. Tex Heart Inst J. 2010;37:361-4.

7. Nakajima T, Iyoda A, Iizasa T, Saitoh Y, Hiroshima K, Fujisawa T. Surgical treatment of a superior sulcus tumor in a patient with idiopathic thrombocytopenic purpura: report of a case. Surg Today. 2005;35:1078-80.

8. Kim YS, Chung JW, Sung BY, Woo YK, Kwon SY. Total hip replacement arthroplasty in patient with idiopathic thrombocytopenic purpura: clinical experience of perioperative management. J Korean Hip Soc. 2008;20:15-8.

9. Nezu M, Oh H, Cho R, Sato H, Ikegami T, Yokota A, Nakaseko C, Nishimura M, Matsuura Y, Morio S, Hiruma K, Nakamura H, Asai T. The combination therapy with vinca-alkaloid slow infusion and cholchicine was effective to refractory idiopathic thrombocytopenic purpura. The patient could receive femoral head replacement in safety. Rinsho Ketsueki. 1996;37:158-60.

10. Hussain SM, Robinson D, Hadden WA. Do uncemented knee bleed more? Prospective, randomised study. J Bone Joint Surg Br. 2006;88(Suppl I):255. 